\title{
QUALITY OF INFORMATION IN “MASSETER BOTOX” VIDEOS ON YOUTUBE: IS IT A SUFFICIENT GUIDE FOR POTENTIAL PATIENTS?
}

\author{
Sezgi Cinel Sahin ${ }^{1}$, Kadriye A. Dere ${ }^{2}$ \\ 'Department of Prosthodontics, Pamukkale University Faculty of Dentistry, Turkey \\ 2Department of Oral and Maxillofacial Surgery, Pamukkale University Faculty of Dentistry, Turkey
}

\begin{abstract}
INTRODUCTION: Recently, the usage of social media for information purposes by patients has gained popularity. ОвјестіVEs: This study aims to evaluate the content of YouTube videos about botulinum toxin injections applied to the masseter muscle.

MATERIAL AND METHODS: A systematic search of YouTube videos was conducted using the key words "masseter Botox", and 102 videos were included in the study. The videos were classified according to type and uploader, and the information content was evaluated. Video information and quality index were used and viewing rates of the videos were also calculated.

RESULTS: Data obtained were analyzed statistically. Most of the videos were found to be information videos (79.4\%), and most of them uploaded by beauty centers (34.3\%). The information content of the educational and information videos was found to be statistically higher than the patient experience videos $(p<0.01)$. When the viewing rates of the videos were examined, it was found that the patient experience videos were observed more, and the interaction rates were statistically higher $(p<0.01)$.

Conclusions: The information content of the masseter Botox-related YouTube videos was found to be insufficient. It is important to overcome this shortcoming with videos with high information content prepared by experts in related fields.
\end{abstract}

KEY WORDs: bruxism, masseter Botox, social media, temporomandibular disorders, YouTube.

J Stoma 2020; 73, 6: 313-325

DOI: https://doi.org/10.5114/jos.2020.102050

\section{INTRODUCTION}

Bruxism is an oral parafunctional habit that affects much of the adult population worldwide, and is considered important by clinicians and researchers because of its negative effects on quality of life [1]. Bruxism is defined as a repetitive jaw-muscle activity characterized by clench- ing or grinding of the teeth and/or by bracing or thrusting of the mandible [2]. Excessive load on the stomatognathic system produced by clenching and grinding causes undesirable conditions, such as pain in the temporomandibular joint, mobility and wear of the teeth, chewing muscle disorders, failure in headaches' restorations, and esthetic problems due to masseter muscle hypertrophy [3].
JOURNAL OF STOMATOLOGY CZASOPISMO STOMATOLOGICZNE
Address For Correspondence: Dr. Sezgi Cinel Sahin, Department of Prosthodontics, Pamukkale University Faculty of Dentistry, Camlaraltı Mahallesi, Suleyman Demirel Cd. No:95, 20160, Denizli, Turkey, e-mail: sezgis@pau.edu.tr

RECEIVED: 27.10.2020 • ACCEPTED: 21.11.2020 • PUBlished: 30.12 .2020 
The main goal in the treatment of bruxism is to defuse the effects of destructive forces caused by clenching and grinding on biological structures and functions. Occlusal adjustments, occlusal splints, surgical approaches, pharmacological therapies, and cognitive-behavioral approaches are used to decrease clinical symptoms, such as tooth wear, facial and temporal pain, and masseteric muscle hypertrophy caused by these forces $[4,5]$. In addition, botulinum toxin (BTX) injections, a conservative, relatively non-invasive and reversible treatment applied to the masseter muscle in the treatment of bruxism, has recently become a popular and preferred treatment option, although it has been an option since $1994[6,7]$.

BTX is an efficacious exotoxin produced by the anaerobic bacterium Clostridium botulinum, which prevents the release of acetylcholine from the cholinergic nerve endings into the neuromuscular junction, thus bringing about the inactivity of muscles or glands [8]. The toxin has seven serotypes, of which the botulinum toxin-A serotype is recommended by the U.S. Food and Drug Administration for the treatment of cosmetic and non-cosmetic medical problems, including head and neck tremors, hemifacial spasms, temporomandibular joint dysfunction, bruxism, chewing myalgias, sialorrhea, hyperhidrosis, and headaches [9].

In today's global world, it has become easy and quick to acquire information by accessing the internet without usage restrictions. When the internet became a platform enabling interaction between users in the early 2000s, its popularity increased, and the number of people accessing the internet from 2000 to 2020 reached over 4.5 billion [10]. In addition to patient-clinician information sharing and interaction, the internet and social media are an important resource for information on dental and health issues, and its use is growing daily [11]. YouTube, one of the most popular video sharing sites, is increasingly used in accessing health information. YouTube is a free-to-access video-sharing website created in 2005 , with approximately 1.5 billion users; each day, 100 million videos are viewed and over 65,000 new videos are uploaded $[12,13]$. Compared to other social media

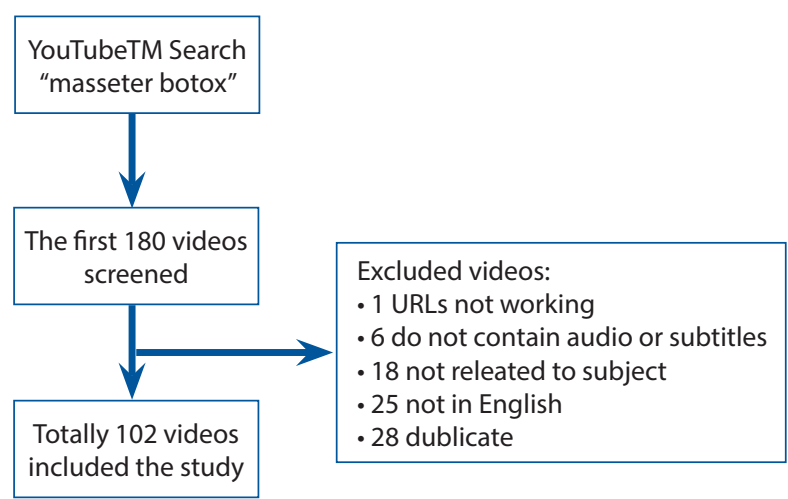

FIGURE 1. Flow chart of the video selection process platforms, it is preferred by those who want to obtain health information due to its ability to provide visual and verbal information [12].

Because social media and internet platforms have grown in popularity in recent years, the number of videos uploaded to the internet by specialists, patients, and private organizations has also increased. Although it is easy to access information, treatment protocols, and comments from the internet, it is necessary to evaluate whether the information and the websites accessed are misleading, false, and biased. The information intensity and complexity make it difficult for users to distinguish the quality and accuracy of video information and can affect the treatment process of patients and, indirectly, clinicians [14]. There are many studies on health topics that evaluate the information flow, origin, and accuracy of related YouTube videos [13, 15-19]. In these studies, the quality of medical content of newly uploaded videos was compared with old videos on a similar subject, and the researchers examined if there was any improvement in terms of video information flow, content, accuracy of the information, and the orientation of patients.

\section{OBJECTIVES}

The interest in BTX injections used in the treatment of muscular symptoms of bruxism in recent years has been increasing rapidly due to its therapeutic and cosmetic effects $[6,7]$. However, few studies analyzed YouTube videos on this subject [19]. For this reason, the present study aims to evaluate the content of current YouTube videos about BTX injections applied to the masseter muscle for the treatment of masseter hypertrophy caused by bruxism.

\section{MATERIAL AND METHODS}

The Google Trends (Google Trends 2019) application was used to determine the most common search term worldwide about BTX applications on the masseter muscle, which was found to be "masseter Botox". A new YouTube (http://www.youtube.com) account was created to prevent the video ranking obtained by searching the key term on YouTube from being affected by old searches. Videos about masseter Botox uploaded through this account up to October 2020 were investigated, without changing YouTube default settings or applying any filters. No ethics committee approval was required for this study, as it was planned to use only public data.

In some studies on the use of YouTube, it was found that approximately $95 \%$ of users view, at most, $60-200$ videos from the search results $[20,21]$. Therefore, in the current study, it was decided to watch the first 180 videos related to the search term "masseter Botox," and the universal resource locators (URLs) of videos were documented. Videos of acceptable quality (240 p and higher), 
in English, and focusing on masseter Botox content were included in the study. Videos that were not in English, did not contain audio or subtitled information, had a broken URLs, duplicates, or were not related to the subject were excluded from the study. This resulted in 120 evaluated videos (Figure 1). The evaluation of the video content and features was done by an oral and maxillofacial surgeon (K.A.D), with a high level of knowledge about masseter hypertrophy and masseter Botox applications.

The methodology used to review the videos in this study was based on previous research [16-18, 22]. All videos were evaluated in detail, and analyzed in terms of video type, video uploaders, information quality of the video content, video quality, and general video information. Regarding the video type, each video was classified as an information, patient experience, or educational video. The uploaders of each video were classified as a specialist doctor, dentist, dental clinic, university/ academy, patient, beauty center, or TV channel.

The information quality of the video content was evaluated by considering current consensus decisions and literature on masseter Botox [23-25]. The content quality of the videos was evaluated according to the following parameters: definition, indication, contraindication, advantages, procedure, complication, prognosis, care and support applications, cost, and specialty branches $[17,22]$. For every video, each of these parameters was scored on a scale of $0-3$ points in line with consensus decisions, with 0 meaning that the video contains no information about the subject or contains misleading information, 1 indicating that the video contains insufficient information about the subject, 2 meaning sufficient information about the video subject, and 3 indicating that the video provides comprehensive information on the subject. The quality of the video was thus evaluated according to the total score obtained by adding the scores from each parameter. A total score of 30 points indicated that the video contained comprehensive and scientifically valid information [22].
The overall quality of each video was evaluated using video information and quality index (VIQI). This evaluation was done using a 5-point Likert-type scale ( $1=$ low quality and flow, 2 = generally poor quality and poor flow, 3 = medium quality and poor flow, $4=$ good quality flow, and 5 = high quality flow), in which the information flow, accuracy of the information, video quality, and level of compliance (sensitivity) between the video title and the content were determined $[17,18]$.

In the last stage, number of views of the videos, period from the uploaded date to the viewing date, likes and dislikes, and running time of the videos were recorded. Using these recorded data, the viewing rates and viewer interactions with the videos were calculated. Two formulas were used in this calculation:

$$
\frac{(\text { number of likes }- \text { number of dislikes })}{\text { total number of views }} \times 100 \%
$$

for calculating viewer interactions with the videos, and

$$
\frac{(\text { number of views })}{\text { number of days since upload }} \times 100 \%
$$

for calculating the viewing rates $[18,22]$.

TABLE 1. Video statistics based on evaluation parameters

\begin{tabular}{|l|c|c|}
\hline Parameter & \multicolumn{1}{c|}{ Average \pm SD } & Min-Max (median) \\
\hline Quality of the video content & $9.25 \pm 4.36$ & $0-21(9)$ \\
\hline VIQI & $17.46 \pm 2.72$ & $8-20(18)$ \\
\hline Interactions index & $1.05 \pm 1.34$ & $-0.07-6.6(0.55)$ \\
\hline Viewing rates & $5,788.09$ & $\begin{array}{c}5.12-214,028.22 \\
(346.73)\end{array}$ \\
\hline
\end{tabular}

$\mathrm{VIQI}$ - video information and quality index, $S D$ - standard deviation

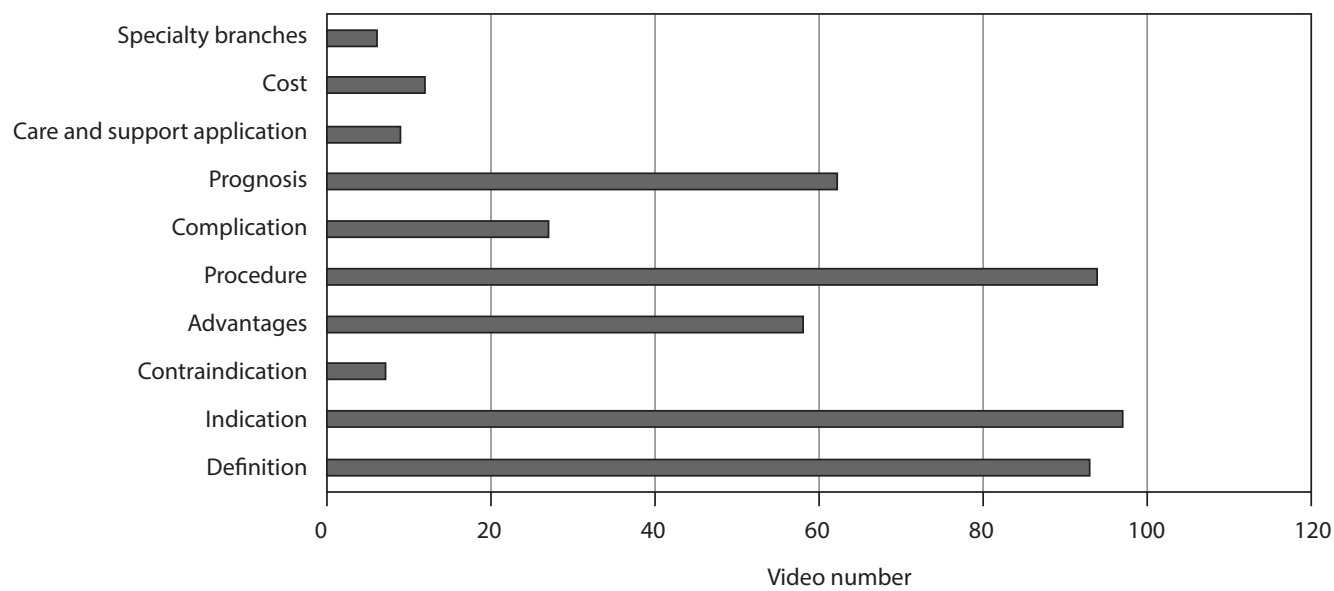

FIGURE 2. Distribution of the titles with the most information in the videos 
Sezgi Cinel Sahin, Kadriye A. Dere

TABLE 2. Comparison of video content quality parameters by video types

\begin{tabular}{|c|c|c|c|c|}
\hline Parameters /Video type & $n$ & Average \pm SD & Min-Max (median) & $p$-value \\
\hline \multicolumn{5}{|l|}{ Definitions } \\
\hline Information & 81 & $1.91 \pm 0.96$ & $0-3(2)$ & \multirow{3}{*}{0.144} \\
\hline Educational & 4 & $2.00 \pm 1.16$ & $1-3(2)$ & \\
\hline Patient's experience & 17 & $1.47 \pm 0.8$ & $0-3(1)$ & \\
\hline \multicolumn{5}{|l|}{ Indications } \\
\hline Information & 81 & $2.33 \pm 0.89$ & $0-3(3)$ & \multirow{3}{*}{0.659} \\
\hline Educational & 4 & $2.25 \pm 0.96$ & $1-3(2.5)$ & \\
\hline Patient's experience & 17 & $2.18 \pm 0.88$ & $0-3(2)$ & \\
\hline \multicolumn{5}{|l|}{ Contraindications } \\
\hline Information & 81 & $0.07 \pm 0.38$ & $0-3(0)$ & \multirow{3}{*}{0.229} \\
\hline Educational & 4 & $0.5 \pm 1.00$ & $0-2(0)$ & \\
\hline Patient's experience & 17 & $0.06 \pm 0.24$ & $0-1(0)$ & \\
\hline \multicolumn{5}{|l|}{ Advantage } \\
\hline Information & 81 & $0.78 \pm 0.78$ & $0-3(1)$ & \multirow{3}{*}{0.978} \\
\hline Educational & 4 & $1.00 \pm 1.41$ & $0-3(0.5)$ & \\
\hline Patient's experience & 17 & $1.00 \pm 1.23$ & $0-3(0)$ & \\
\hline \multicolumn{5}{|l|}{ Procedure } \\
\hline Information & 81 & $1.84 \pm 0.93$ & $0-3(2)$ & \multirow{3}{*}{$0.039^{*}$} \\
\hline Educational & 4 & $2.5 \pm 0.58$ & $2-3(2.5)$ & \\
\hline Patient's experience & 17 & $1.41 \pm 0.71$ & $0-3(1)$ & \\
\hline \multicolumn{5}{|l|}{ Complication } \\
\hline Information & 81 & $0.51 \pm 0.98$ & $0-3(0)$ & \multirow{3}{*}{$0.023^{*}$} \\
\hline Educational & 4 & $2.25 \pm 1.5$ & $0-3(3)$ & \\
\hline Patient's experience & 17 & $0.47 \pm 0.94$ & $0-3(0)$ & \\
\hline \multicolumn{5}{|l|}{ Prognosis } \\
\hline Information & 81 & $1.28 \pm 1.3$ & $0-3(1)$ & \multirow{3}{*}{0.380} \\
\hline Educational & 4 & $1.25 \pm 1.26$ & $0-3(1)$ & \\
\hline Patient's experience & 17 & $1.71 \pm 1.16$ & $0-3(2)$ & \\
\hline \multicolumn{5}{|l|}{ Care and support applications } \\
\hline Information & 81 & $0.09 \pm 0.42$ & $0-3(0)$ & \multirow{3}{*}{$0.005^{* *}$} \\
\hline Educational & 4 & $0.0 \pm 0.0$ & $0-0(0)$ & \\
\hline Patient's experience & 17 & $0.53 \pm 0.94$ & $0-3(0)$ & \\
\hline \multicolumn{5}{|l|}{ Cost } \\
\hline Information & 81 & $0.14 \pm 0.49$ & $0-3(0)$ & \multirow{3}{*}{0.103} \\
\hline Educational & 4 & $0.75 \pm 1.5$ & $0-3(0)$ & \\
\hline Patient's experience & 17 & $0.71 \pm 1.31$ & $0-3(0)$ & \\
\hline \multicolumn{5}{|l|}{ Specialty branches } \\
\hline Information & 81 & $0.06 \pm 0.24$ & $0-1(0)$ & \multirow{3}{*}{0.159} \\
\hline Educational & 4 & $0.25 \pm 0.5$ & $0-1(0)$ & \\
\hline Patient's experience & 17 & $0.0 \pm 0.0$ & $0-0(0)$ & \\
\hline \multicolumn{5}{|l|}{ Total } \\
\hline Information & 81 & $9.01 \pm 4.16$ & $0-20(9)$ & \multirow{3}{*}{0.458} \\
\hline Educational & 4 & $12.75 \pm 6.24$ & $6-21(12)$ & \\
\hline Patient's experience & 17 & $9.53 \pm 4.73$ & 2-16(11) & \\
\hline
\end{tabular}

Kruskal-Wallis test: ${ }^{*} p<0.05,{ }^{* *} p<0.01 ; S D$ - standard deviation 
TABLE 3. Comparison of video information and quality index (VIQI) interaction index and viewing rates data by video types

\begin{tabular}{|c|c|c|c|c|}
\hline Parameters /Video type & $n$ & Average \pm SD & Min-Max (median) & $p$-value \\
\hline \multicolumn{5}{|l|}{ Information flow } \\
\hline Information & 81 & $3.99 \pm 1.19$ & $1-5(4)$ & \multirow{3}{*}{$0.013^{*}$} \\
\hline Educational & 4 & $3.75 \pm 0.96$ & $3-5(3.5)$ & \\
\hline Patient's experience & 17 & $3.24 \pm 1.03$ & $1-5(3)$ & \\
\hline \multicolumn{5}{|l|}{ Accuracy of information } \\
\hline Information & 81 & $4.69 \pm 0.9$ & $1-5(5)$ & \multirow{3}{*}{$0.001^{* *}$} \\
\hline Educational & 4 & $4.75 \pm 0.5$ & $4-5(5)$ & \\
\hline Patient's experience & 17 & $3.71 \pm 1.4$ & $1-5(4)$ & \\
\hline \multicolumn{5}{|l|}{ Video quality } \\
\hline Information & 81 & $4.36 \pm 0.93$ & $1-5(5)$ & \multirow{3}{*}{0.362} \\
\hline Educational & 4 & $4.25 \pm 0.5$ & $4-5(4)$ & \\
\hline Patient's experience & 17 & $4.59 \pm 0.8$ & $2-5(5)$ & \\
\hline \multicolumn{5}{|c|}{ Level of compliance (sensitivity) } \\
\hline Information & 81 & $4.74 \pm 0.61$ & $2-5(5)$ & \multirow{3}{*}{0.296} \\
\hline Educational & 4 & $5.0 \pm 0.0$ & $5-5(5)$ & \\
\hline Patient's experience & 17 & $4.35 \pm 1.22$ & $1-5(5)$ & \\
\hline \multicolumn{5}{|l|}{ VIQI total score } \\
\hline Information & 81 & $17.78 \pm 2.47$ & $9-20(18)$ & \multirow{3}{*}{0.062} \\
\hline Educational & 4 & $17.75 \pm 1.71$ & $16-20(17.5)$ & \\
\hline Patient's experience & 17 & $15.88 \pm 3.53$ & $8-20(17)$ & \\
\hline \multicolumn{5}{|l|}{ Interaction index } \\
\hline Information & 81 & $0.96 \pm 1.28$ & $-0.07-6.6(0.45)$ & \multirow{3}{*}{0.223} \\
\hline Educational & 4 & $1.72 \pm 3.03$ & $0-6.25(0.31)$ & \\
\hline Patient's experience & 17 & $1.34 \pm 1.08$ & $0-3.32(1.16)$ & \\
\hline \multicolumn{5}{|l|}{ Viewing rates } \\
\hline Information & 81 & $1,420.87 \pm 2,894.39$ & 5.12-16,929.43 (296.8) & \multirow{3}{*}{$0.003 * *$} \\
\hline Educational & 4 & $221.58 \pm 152.32$ & $87.1-440.55(179.34)$ & \\
\hline Patient's experience & 17 & $29,288.8 \pm 56,854.95$ & $\begin{array}{c}13.83-214,028.22 \\
(3,780.84)\end{array}$ & \\
\hline
\end{tabular}

Kruskal-Wallis test: ${ }^{*} p<0.05,{ }^{* *} p<0.01 ; S D$ - standard deviation

\section{STATISTICAL ANALYSIS}

Number Cruncher Statistical System 2007 (NCSS, Kaysville, Utah, USA) was used for a statistical analysis. The descriptive statistical methods and distribution of the data were evaluated with Shapiro-Wilk test. Kruskal-Wallis analysis was applied to compare three or more groups, which did not show a normal distribution of the quantitative data, and Mann-Whitney $U$ analysis was used to compare two groups that did not show a normal distribution. Spearman's correlation test was utilized to determine relationships among the quantitative data. The significance was evaluated at $p<0.01$ and $p<0.05$ levels.

\section{RESULTS}

When the 102 YouTube videos watched were classified by the video type, it was determined that $79.4 \%$ $(n=81)$ of the videos were information videos, $3.9 \%$ $(n=4)$ were educational videos, and $16.7 \%(n=17)$ were patients' experience videos. Further, when the distribution of videos in terms of the uploader was examined, it was found that most of the videos were uploaded by beauty centers - 34.3\% $(n=35)$, followed by specialist doctors $-21.6 \%(n=22)$, dental clinics and patients both $14.7 \%(n=15)$, universities $-6.9 \%(n=7)$, and dentists and TV channels both $3.9 \%(n=4)$. 
TABLE 4. Comparison of video content quality parameters by video uploaders

\begin{tabular}{|c|c|c|c|c|}
\hline Parameters/Video uploader & $n$ & Average \pm SD & Min-Max (median) & $p$-value \\
\hline \multicolumn{5}{|l|}{ Definitions } \\
\hline Dental clinic & 15 & $2 \pm 0.93$ & $0-3(2)$ & \multirow{7}{*}{0.133} \\
\hline Dentist & 4 & $1.5 \pm 1.29$ & $0-3(1.5)$ & \\
\hline Beauty center & 35 & $1.86 \pm 0.88$ & $0-3(2)$ & \\
\hline Patient & 15 & $1.53 \pm 0.83$ & $0-3(1)$ & \\
\hline TV channel & 4 & $2.5 \pm 0.58$ & $2-3(2.5)$ & \\
\hline Specialist doctor & 22 & $1.64 \pm 1.09$ & $0-3(2)$ & \\
\hline University/academia & 7 & $2.57 \pm 0.79$ & $1-3(3)$ & \\
\hline \multicolumn{5}{|l|}{ Indications } \\
\hline Dental clinic & 15 & $2.4 \pm 0.74$ & $1-3(3)$ & \multirow{7}{*}{0.148} \\
\hline Dentist & 4 & $1.75 \pm 1.26$ & $0-3(2)$ & \\
\hline Beauty center & 35 & $2.34 \pm 0.87$ & $1-3(3)$ & \\
\hline Patient & 15 & $2.13 \pm 0.92$ & $0-3(2)$ & \\
\hline TV channel & 4 & $3.0 \pm 0.0$ & $3-3(3)$ & \\
\hline Specialist doctor & 22 & $2.09 \pm 1.02$ & $0-3(2)$ & \\
\hline University/academia & 7 & $2.86 \pm 0.38$ & $2-3(3)$ & \\
\hline \multicolumn{5}{|l|}{ Contraindication } \\
\hline Dental clinic & 15 & $0.0 \pm 0.0$ & $0-0(0)$ & \multirow{7}{*}{0.837} \\
\hline Dentist & 4 & $0.0 \pm 0.0$ & $0-0(0)$ & \\
\hline Beauty center & 35 & $0.11 \pm 0.53$ & $0-3(0)$ & \\
\hline Patient & 15 & $0.07 \pm 0.26$ & $0-1(0)$ & \\
\hline TV channel & 4 & $0.0 \pm 0.0$ & $0-0(0)$ & \\
\hline Specialist doctor & 22 & $0.09 \pm 0.29$ & $0-1(0)$ & \\
\hline University/academia & 7 & $0.29 \pm 0.76$ & $0-2(0)$ & \\
\hline \multicolumn{5}{|l|}{ Advantages } \\
\hline Dental clinic & 15 & $1.07 \pm 0.96$ & $0-3(1)$ & \multirow{7}{*}{0.441} \\
\hline Dentist & 4 & $0.75 \pm 0.96$ & $0-2(0.5)$ & \\
\hline Beauty center & 35 & $0.66 \pm 0.73$ & $0-2(1)$ & \\
\hline Patient & 15 & $1.07 \pm 1.28$ & $0-3(0)$ & \\
\hline TV channel & 4 & $1.5 \pm 0.58$ & $1-2(1.5)$ & \\
\hline Specialist doctor & 22 & $0.64 \pm 0.58$ & $0-2(1)$ & \\
\hline University/academia & 7 & $0.86 \pm 1.22$ & $0-3(0)$ & \\
\hline \multicolumn{5}{|l|}{ Procedure } \\
\hline Dental clinic & 15 & $1.73 \pm 0.96$ & $0-3(2)$ & \multirow{7}{*}{$0.019 *$} \\
\hline Dentist & 4 & $1.0 \pm 0.82$ & $0-2(1)$ & \\
\hline Beauty center & 35 & $2.0 \pm 0.91$ & $0-3(2)$ & \\
\hline Patient & 15 & $1.53 \pm 0.64$ & $1-3(1)$ & \\
\hline TV channel & 4 & $1.0 \pm 0.82$ & $0-2(1)$ & \\
\hline Specialist doctor & 22 & $1.73 \pm 0.94$ & $0-3(2)$ & \\
\hline University/academia & 7 & $2.57 \pm 0.54$ & $2-3(3)$ & \\
\hline
\end{tabular}


TABLE 4. Cont.

\section{Parameters/Video uploader}

$n$

Average \pm SD

Min-Max (median)

p-value

Complications

\begin{tabular}{l|c|c|c|}
\hline Dental clinic & 15 & $0.93 \pm 1.39$ & $0-3(0)$ \\
\hline Dentist & 4 & $0.0 \pm 0.0$ & $0-0(0)$ \\
\hline Beauty center & 35 & $0.49 \pm 0.89$ & $0-3(0)$ \\
\hline Patient & 15 & $0.53 \pm 0.99$ & $0-3(0)$ \\
\hline TV channel & 4 & $0.0 \pm 0.0$ & $0-0(0)$ \\
\hline Specialist doctor & 22 & $0.45 \pm 0.91$ & $0-3(0)$ \\
\hline University/academia & 7 & $1.29 \pm 1.6$ & $0-3(0)$ \\
\hline
\end{tabular}

Prognosis

\begin{tabular}{l|c|c|c|}
\hline Dental clinic & 15 & $1.4 \pm 1.24$ & $0-3(1)$ \\
\hline Dentist & 4 & $0.25 \pm 0.5$ & $0-1(0)$ \\
\hline Beauty center & 35 & $1.43 \pm 1.27$ & $0-3(1)$ \\
\hline Patient & 15 & $1.8 \pm 1.21$ & $0-3(2)$ \\
\hline TV channel & 4 & $0.75 \pm 1.5$ & $0-3(0)$ \\
\hline Specialist doctor & 22 & $1.14 \pm 1.32$ & $0-3(0)$ \\
\hline University/academia & 7 & $1.57 \pm 1.4$ & $0-3(1)$ \\
\hline
\end{tabular}

Care and support applications

\begin{tabular}{l|c|c|c|}
\hline Dental clinic & 15 & $0.0 \pm 0.0$ & $0-0(0)$ \\
\hline Dentist & 4 & $0.0 \pm 0.0$ & $0-0(0)$ \\
\hline Beauty center & 35 & $0.11 \pm 0.4$ & $0-2(0)$ \\
\hline Patient & 15 & $0.6 \pm 0.99$ & $0-3(0)$ \\
\hline TV channel & 4 & $0.0 \pm 0.0$ & $0-0(0)$ \\
\hline Specialist doctor & 22 & $0.0 \pm 0.0$ & $0-0(0)$ \\
\hline University/academia & 7 & $0.43 \pm 1.13$ & $0-3(0)$ \\
\hline
\end{tabular}

Cost

\begin{tabular}{l|c|c|c|}
\hline Dental clinic & 15 & $0.27 \pm 0.8$ & $0-3(0)$ \\
\hline Dentist & 4 & $0.0 \pm 0.0$ & $0-0(0)$ \\
\hline Beauty center & 35 & $0.2 \pm 0.63$ & $0-3(0)$ \\
\hline Patient & 15 & $0.8 \pm 1.37$ & $0-3(0)$ \\
\hline TV channel & 4 & $0.0 \pm 0.0$ & $0-0(0)$ \\
\hline Specialist doctor & 22 & $0.14 \pm 0.47$ & $0-2(0)$ \\
\hline University/academia & 7 & $0.0 \pm 0.0$ & $0-0(0)$
\end{tabular}

Specialty branches

\begin{tabular}{l|c|c|c|}
\hline Dental clinic & 15 & $0.07 \pm 0.26$ & $0-1(0)$ \\
\hline Dentist & 4 & $0.25 \pm 0.5$ & $0-1(0)$ \\
\hline Beauty center & 35 & $0.03 \pm 0.17$ & $0-1(0)$ \\
\hline Patient & 15 & $0.0 \pm 0.0$ & $0.024^{*}$ \\
\hline TV channel & 4 & $0.25 \pm 0.5$ & $0-1(0)$ \\
\hline Specialist doctor & 22 & $0.0 \pm 0.0$ & $0-0(0)$ \\
\hline University/academia & 7 & $0.29 \pm 0.49$ & $0-1(0)$ \\
\hline
\end{tabular}


TABLE 4. Cont

\begin{tabular}{|c|c|c|c|c|}
\hline Parameters/Video uploader & $n$ & Average \pm SD & Min-Max (median) & $p$-value \\
\hline \multicolumn{5}{|l|}{ Total } \\
\hline Dental clinic & 15 & $9.87 \pm 3.52$ & $2-15(11)$ & \multirow{7}{*}{0.230} \\
\hline Dentist & 4 & $5.5 \pm 3.42$ & $2-10(5)$ & \\
\hline Beauty center & 35 & $9.23 \pm 4.03$ & 3-15 (9) & \\
\hline Patient & 15 & $10.07 \pm 4.79$ & $2-16(11)$ & \\
\hline TV channel & 4 & $9.0 \pm 2.71$ & $7-13(8)$ & \\
\hline Specialist doctor & 22 & $7.91 \pm 4.46$ & $0-16(8)$ & \\
\hline University/academia & 7 & $12.71 \pm 5.77$ & $6-21(11)$ & \\
\hline
\end{tabular}

Kruskal-Wallis test: ${ }^{*} p<0.05,{ }^{* *} p<0.01 ; S D$ - standard deviation

Average, maximum, minimum, and median values of all evaluations in the study were calculated. The calculations related to the information quality of the video content, the VIQI, viewer interaction index, and viewing rates are summarized in Table 1 . When the information quality of the video content was evaluated, the scores ranged from 0 to 21 , and none of the videos received full points in terms of information content (Table 1). The distribution of the titles with the most information in the videos is shown in Figure 2.

When the video content quality was evaluated, it was found that procedure values of information and educational videos were lower than the patients' experience videos $(p<0.01)$. Further, the complication values of the educational videos were statistically significant compared to the patients' experience and information videos $(p<0.01)$. In addition, the values of care and support practices of the patients' experience videos were statistically significant compared to the information videos $(p<0.01)$ (Table 2).

The statistical comparison of parameters examined within the scope of VIQI by video type is summarized in Table 3. The information flow and information accuracy values of the information videos were found to be statistically significant compared to the patients' experience videos $(p<0.01)$. The viewing rates of the patients' experience videos were higher than the information and education videos, which was found to be statistically significant $(p<0.01)$ (Table 3).

There was a statistically significant difference between the procedure values according to the video uploaders $(p<0.05)$. The information content of the video uploaded by the university/ academy on the procedure was higher than all the other uploaders, except for the beauty centers, which was found to be statistically significant $(p<0.01)$. There was also a statistically significant difference between care and support application values according to the video uploader $(p<0.05)$. It was statistically significantly found that the information content values related to the care and support application of the videos uploaded by patients were higher than the videos uploaded by spe- cialist doctors $(p<0.01)$. In addition, there were statistical differences among the videos in terms of containing information about the specialist branches according to the video uploaders $(p<0.05)$. Accordingly, the information content of videos uploaded by specialist doctors was found to be statistically lower than videos uploaded by dentists and TV channels. The videos uploaded by universities/ academies were found to be statistically higher in terms of information compared to videos uploaded by beauty centers, patients, and specialist doctors $(p<0.01)$ (Table 4).

The statistical comparison of parameters examined within the scope of VIQI for video uploaders are summarized in Table 5. It was statistically significantly observed that the information accuracy of videos uploaded by patients was lower than that of videos uploaded by dental clinics, beauty centers, specialist doctors, and universities/academies $(p<0.01)$. On the other hand, the interaction index of videos uploaded by patients was found to be statistically significant compared to the videos uploaded by dental clinics, beauty centers, and TV channels $(p<0.01)$. In addition, the rate of viewing videos uploaded by patients compared to other groups was found to be statistically significant $(p<0.01)$. Also, the viewing rates of videos uploaded by dental clinics were found to be statistically significant compared to the videos uploaded by beauty centers and specialist doctors $(p<0.01)$ (Table 5). The relationships between all parameters used in the study were compared, and are presented in Table 6. As a result of this comparison, it was determined that there was a positive and highly significant relationship between the definition and indication parameters, the prognosis and the total score of all evaluation criteria, and between the information flow and the VIQI total score (Table 6).

\section{DISCUSSION}

In the long-term, the parafunctional activities of chewing muscles, such as bruxism, cause damage to patients in dental and temporomandibular joints, and 
TABLE 5. Comparison of video information and quality index (VIQI) interaction index and viewing rates data by video uploaders

\begin{tabular}{|c|c|c|c|c|}
\hline Parameters/Video uploader & $n$ & Average \pm SD & Min-Max (median) & $p$-value \\
\hline \multicolumn{5}{|l|}{ Information flow } \\
\hline Dental clinic & 15 & $4.13 \pm 0.92$ & $2-5(4)$ & \multirow{7}{*}{$0.034^{*}$} \\
\hline Dentist & 4 & $2.75 \pm 1.71$ & $1-5(2.5)$ & \\
\hline Beauty center & 35 & $3.89 \pm 1.21$ & $1-5(4)$ & \\
\hline Patient & 15 & $3.2 \pm 1.08$ & $1-5(3)$ & \\
\hline TV channel & 4 & $4.75 \pm 0.5$ & $4-5(5)$ & \\
\hline Specialist doctor & 22 & $3.91 \pm 1.23$ & $1-5(4)$ & \\
\hline University/academia & 7 & $4.43 \pm 0.79$ & $3-5(5)$ & \\
\hline \multicolumn{5}{|l|}{ Accuracy of the information } \\
\hline Dental clinic & 15 & $4.8 \pm 0.56$ & $3-5(5)$ & \multirow{7}{*}{$0.001^{* *}$} \\
\hline Dentist & 4 & $3.25 \pm 2.06$ & $1-5(3.5)$ & \\
\hline Beauty center & 35 & $4.83 \pm 0.51$ & $3-5(5)$ & \\
\hline Patient & 15 & $3.6 \pm 1.45$ & $1-5(4)$ & \\
\hline TV channel & 4 & $4.75 \pm 0.5$ & $4-5(5)$ & \\
\hline Specialist doctor & 22 & $4.55 \pm 1.18$ & $1-5(5)$ & \\
\hline University/academia & 7 & $5.0 \pm 0.0$ & $5-5(5)$ & \\
\hline \multicolumn{5}{|l|}{ Video quality } \\
\hline Dental clinic & 15 & $4.33 \pm 1.11$ & $1-5(5)$ & \multirow{7}{*}{0.134} \\
\hline Dentist & 4 & $4.25 \pm 0.96$ & $3-5(4.5)$ & \\
\hline Beauty center & 35 & $4.54 \pm 0.74$ & $2-5(5)$ & \\
\hline Patient & 15 & $4.6 \pm 0.83$ & $2-5(5)$ & \\
\hline TV channel & 4 & $3.25 \pm 0.96$ & $2-4(3.5)$ & \\
\hline Specialist doctor & 22 & $4.32 \pm 0.95$ & $2-5(5)$ & \\
\hline University/academia & 7 & $4.29 \pm 0.76$ & $3-5(4)$ & \\
\hline \multicolumn{5}{|l|}{ Level of compliance (sensitivity) } \\
\hline Dental clinic & 15 & $4.87 \pm 0.52$ & $3-5(5)$ & \multirow{7}{*}{0.055} \\
\hline Dentist & 4 & $3.75 \pm 1.26$ & $2-5(4)$ & \\
\hline Beauty center & 35 & $4.71 \pm 0.57$ & $3-5(5)$ & \\
\hline Patient & 15 & $4.33 \pm 1.29$ & $1-5(5)$ & \\
\hline TV channel & 4 & $5.0 \pm 0.0$ & $5-5(5)$ & \\
\hline Specialist doctor & 22 & $4.77 \pm 0.53$ & $3-5(5)$ & \\
\hline University/academia & 7 & $5.0 \pm 0.0$ & $5-5(5)$ & \\
\hline \multicolumn{5}{|l|}{ VIQI total } \\
\hline Dental clinic & 15 & $18.13 \pm 2.26$ & $13-20(19)$ & \multirow{7}{*}{0.153} \\
\hline Dentist & 4 & $14.0 \pm 5.23$ & $9-19(14)$ & \\
\hline Beauty center & 35 & $17.97 \pm 2.08$ & $11-20(18)$ & \\
\hline Patient & 15 & $15.73 \pm 3.73$ & $8-20(17)$ & \\
\hline TV channel & 4 & $17.75 \pm 0.5$ & $17-18(18)$ & \\
\hline Specialist doctor & 22 & $17.55 \pm 2.41$ & $11-20(18)$ & \\
\hline University/academia & 7 & $18.71 \pm 1.11$ & $17-20(19)$ & \\
\hline
\end{tabular}


TABLE 5. Cont.

\begin{tabular}{|c|c|c|c|c|}
\hline Parameters/Video uploader & $n$ & Average \pm SD & Min-Max (median) & $p$-value \\
\hline \multicolumn{5}{|l|}{ Interaction index } \\
\hline Dental clinic & 15 & $1.0 \pm 1.63$ & $0-5(0.22)$ & \multirow{7}{*}{$0.032 *$} \\
\hline Dentist & 4 & $1.29 \pm 1.32$ & $0-3.13(1.03)$ & \\
\hline Beauty center & 35 & $0.72 \pm 1.18$ & $-0.07-6.6(0.38)$ & \\
\hline Patient & 15 & $1.52 \pm 1.04$ & $0-3.32(1.44)$ & \\
\hline TV channel & 4 & $0.42 \pm 0.18$ & $0.2-0.63(0.42)$ & \\
\hline Specialist doctor & 22 & $0.98 \pm 0.92$ & $0-4.28(0.84)$ & \\
\hline University/academia & 7 & $2.34 \pm 2.54$ & $0.27-6.25(1.17)$ & \\
\hline \multicolumn{5}{|l|}{ Viewing rates } \\
\hline Dental clinic & 15 & $658.5 \pm 1,750.99$ & $13.59-6,888.57$ (115.57) & \multirow{7}{*}{$0.001^{* *}$} \\
\hline Dentist & 4 & $216.93 \pm 267.98$ & $11-576.56(140.08)$ & \\
\hline Beauty center & 35 & $1,379.87 \pm 3,179.61$ & $5.12-16,929.43(332.14)$ & \\
\hline Patient & 15 & $33,467.45 \pm 59,827.72$ & $\begin{array}{c}134.29-214,028.22 \\
(4,127.16)\end{array}$ & \\
\hline TV channel & 4 & $1,398.11 \pm 1,484.93$ & $\begin{array}{c}63.86-2,736.43 \\
(1,396.08)\end{array}$ & \\
\hline Specialist doctor & 22 & $1,840.26 \pm 3,079.62$ & $26.34-11,779.67(340.22)$ & \\
\hline University/academia & 7 & $1,562.07 \pm 3,381.42$ & 24.07-9,196.11 (177.78) & \\
\hline
\end{tabular}

Kruskal-Wallis test: ${ }^{*} p<0.05,{ }^{* *} p<0.01 ; S D$ - standard deviation

the treatment of this condition involves changing the existing muscle function and helping to manage parafunctional habits [4]. Although doctors give information to patients about BTX-A treatment, patients usually need additional information and they often use the internet for this reason.

There are many YouTube analysis studies on health issues [13, 15-18], but only one study on BTX applications in the treatment of bruxism has been identified [19]. However, in this present study, it was detected that the parameters used in the analysis of the information content of the videos were limited, and relationships between the parameters were not evaluated. In addition, key words, evaluation criteria, and evaluation time used in the study were different. Due to growing interest in the treatment of masseter hypertrophy caused by bruxism with BTX, the present study intended to analyze the information content and quality of current videos.

It was found that almost all the videos contain information in terms of the definition, indication, and procedure. However, in line with other studies $[15,22]$, it was found that the number of videos providing information about complications, contraindications, cost, care, and support applications was very low. It is critical to overcome the lack of information on these issues, especially considering directing patients correctly, performing flawless applications, and determining an appropriate treatment $[4,6,15,22]$.

Over the next few years, it is believed that the internet will become the primary source of information gathering; therefore, it is essential to ensure access to quality videos [15-19]. However, many studies evaluating the quality of health-related YouTube videos have reported poor video information content quality [17, 19, 22, 26, 27]. In our study, similar to these findings, no video scored fully in terms of information content. The video with the highest information content was rated 21 points over 30 points. The lack of high-quality videos may be related to the videos originating from different professional groups as well as beauty centers and bloggers due to the increased interest in BTX for aesthetic reasons [22]. Conversely, in a similar study in the literature, it was reported that most videos contain high information for patients [19]. It is thought that these differences between studies occurred because the current study included far more parameters evaluating the information content than the other research.

There was no conclusion whether contents of the analyzed videos were misleading. However, it was observed that there were inconsistencies among the videos, especially relating to the BTX application dose. The reason for these differences could be because there is no obligation to provide any scientific reference for videos uploaded to platforms such as YouTube $[17,19]$. In this examination, it was determined that only a few videos uploaded for educational purposes presented references regarding information provided.

In many studies on YouTube, it has been found that most of the videos were uploaded by patients [28, 29]. Unlike these studies, the current study observed that the number of videos uploaded by beauty centers was 


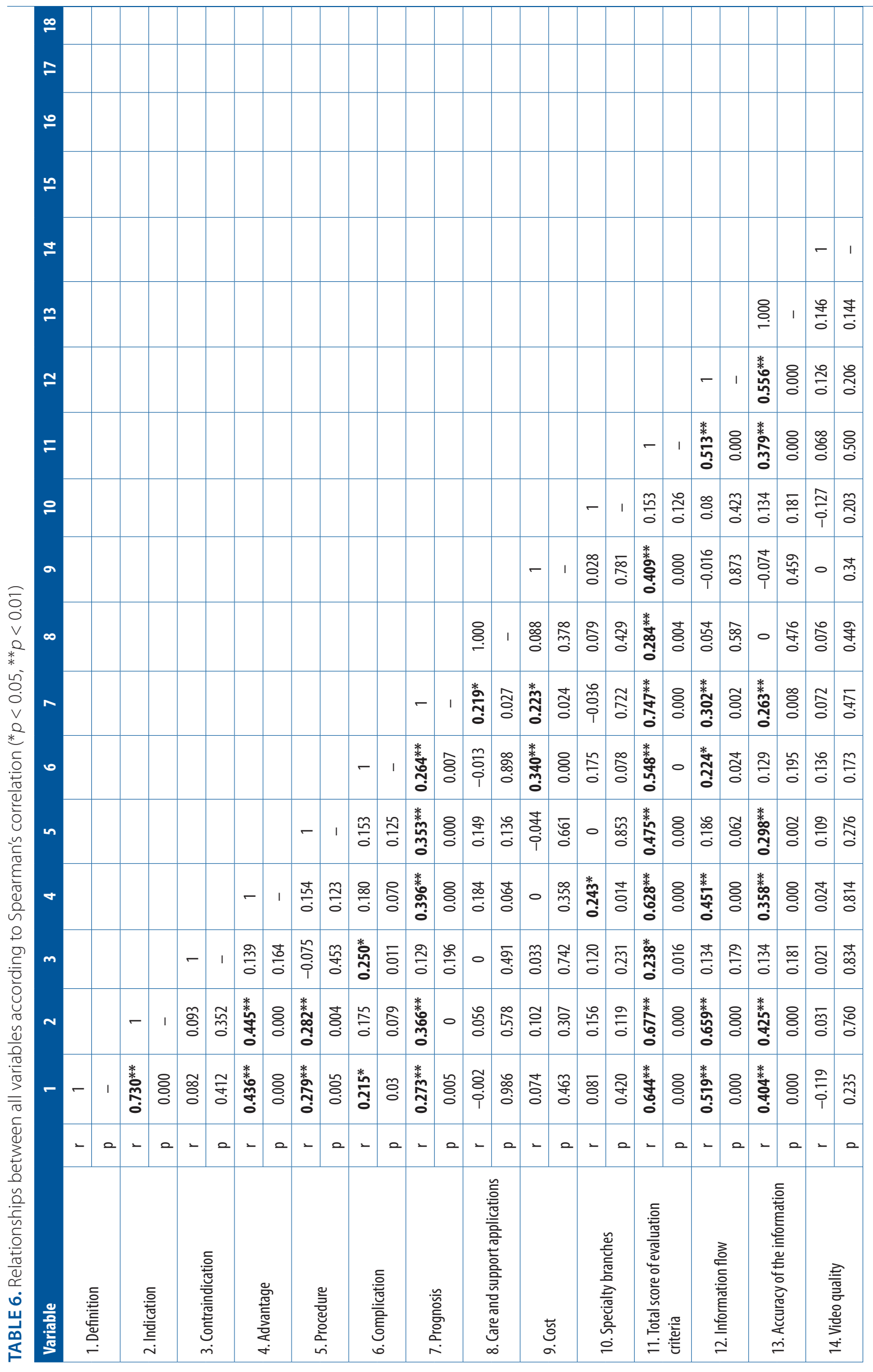




\begin{tabular}{|c|c|c|c|c|c|c|c|c|}
\hline$\stackrel{\infty}{\simeq}$ & & & & & & - & 1 & \multirow{16}{*}{ 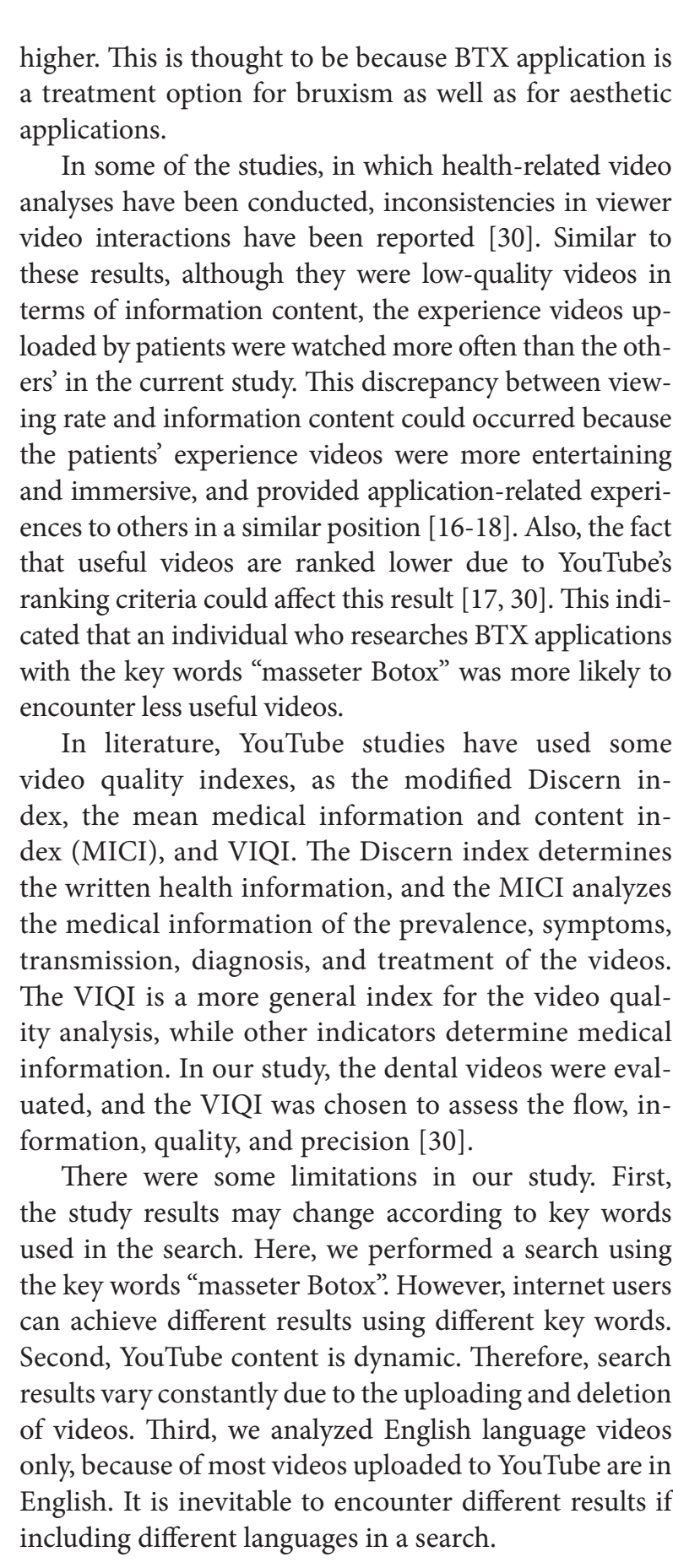 } \\
\hline$=$ & & & & - & 1 & $\bar{\delta}$ & 公 & \\
\hline 누 & & $\stackrel{\text { ¿ }}{-}$ & & 㔛 & $\begin{array}{l}\tilde{O} \\
\tilde{\alpha} \\
0 \\
0\end{array}$ & $\begin{array}{l}\hat{\tilde{O}} \\
\dot{O}\end{array}$ & 彔 & \\
\hline & - & 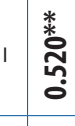 & 号 & 离 & స్ట్ర & $\begin{array}{l}\text { ठิ } \\
\text { iे }\end{array}$ & $\underset{\sigma}{\stackrel{c}{\sigma}}$ & \\
\hline & & 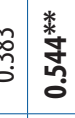 & ఫ̊ & $\begin{array}{l}\overline{0} \\
\dot{1} \\
\end{array}$ & $\frac{\infty}{0}$ & $\stackrel{\infty}{\circ}$ & $\begin{array}{l}\bar{\sigma} \\
\text { o. } \\
0\end{array}$ & \\
\hline & 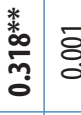 & S. & 号 & 总 & ర్ర్ర & 0 & $\frac{⿱}{8}$ & \\
\hline & & 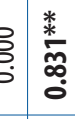 & 응 & 0 & 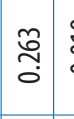 & $\begin{array}{l}0 \\
\overline{0} \\
\bar{i}\end{array}$ & 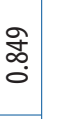 & \\
\hline & 絭 & 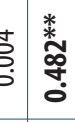 & 0 & 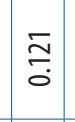 & సి & 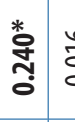 & $\begin{array}{l}0 \\
\stackrel{0}{0} \\
0\end{array}$ & \\
\hline 은 & 0 & $\delta_{\delta} \bar{\delta}$ & 音 & $\frac{\check{\sigma}}{\circ}$ & $\stackrel{0}{0}$ & $\underset{\frac{m}{c}}{\stackrel{c}{i}}$ & 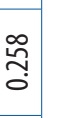 & \\
\hline & $\begin{array}{l}\stackrel{2}{\sigma} \\
\sigma\end{array}$ & o̊ & $\begin{array}{c}\text { 妾 } \\
0 \\
0\end{array}$ & $\underset{\Xi}{\Xi}$ & 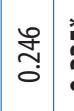 & 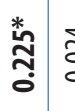 & 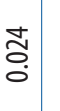 & \\
\hline & 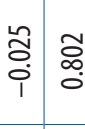 & $\begin{array}{l}\text { 苔 } \\
\text { O }\end{array}$ & 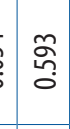 & 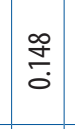 & $\frac{\tilde{m}}{\dot{\sigma}}$ & 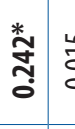 & $\begin{array}{l}n \\
\bar{\delta} \\
0 \\
0\end{array}$ & \\
\hline & $\frac{8}{\circ}$ & $\underset{\text { 絭 }}{\stackrel{2}{0}}$ & $\bar{\delta}$ & $\tilde{\Xi}$ & 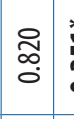 & \begin{tabular}{l} 
ֻ. \\
\multirow{2}{*}{} \\
0
\end{tabular} & $\begin{array}{l}\bar{\sigma} \\
0 \\
0\end{array}$ & \\
\hline & $\stackrel{\cong}{\circ}$ & 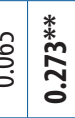 & 茴 & $\frac{\bar{\sigma}}{0}$ & $\overline{\tilde{m}}$ & 容 & 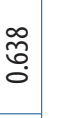 & \\
\hline & 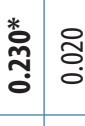 & 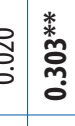 & ठ̊. & $\begin{array}{l}\tilde{O} \\
0 \\
i \\
1\end{array}$ & 命 & $\begin{array}{l}8 \\
0 \\
0\end{array}$ & 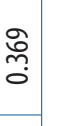 & \\
\hline & 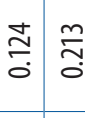 & $\begin{array}{l}\frac{1}{2} \\
\stackrel{3}{\circ} \\
\end{array}$ & 0 & $\mid \begin{array}{l}\tilde{o} \\
\tilde{o} \\
0\end{array}$ & 导 & 岁 & 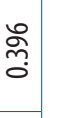 & \\
\hline$m$ & $\begin{array}{l}\underset{Z}{0} \\
0\end{array}$ & \begin{tabular}{c}
$n$ \\
\hdashline
\end{tabular} & $\frac{\text { 导 }}{0}$ & $\begin{array}{c}0 \\
\frac{0}{0} \\
i\end{array}$ & 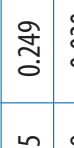 & $\begin{array}{l}\tilde{o} \\
\substack{0 \\
i}\end{array}$ & $\begin{array}{l}\text { o. } \\
\text { o. }\end{array}$ & \\
\hline
\end{tabular}

\section{CONCLUSIONS}

YouTube can be an important source of patient information, but the information content quality of the masseter Botox-related videos examined was found to be low. YouTube and similar platforms to be health-related information sources, healthcare professionals, and educational institutions, such as universities or academies, must undertake initiatives. Besides having experts or institutions upload videos with up-to-date information, reviewing the uploaded videos in terms of content is also valuable for directing patients. Further studies are needed to examine the usefulness of YouTube and other 
digital platforms, and to test its usability as a pre-clinical information platform for informing patients about BTX applications for bruxism-induced masseter hypertrophy.

\section{ACKNOWLEDGEMENTS}

We would like to thank Hande Emir for the statistical analyses.

\section{CONFLICT OF INTEREST}

The authors declare no potential conflicts of interest with respect to the research, authorship, and/or publication of this article.

\section{References}

1. Johansson A, Omar R, Carlsson GE. Bruxism and prosthetic treatment: a critical review. J Prosthodont Res 2011; 55: 127-136.

2. Lobbezoo F, Ahlberg J, Glaros AG, et al. Bruxism defined and graded: an international consensus. J Oral Rehabil 2013; 40: 2-4.

3. Lobbezoo F, Brouwers JEIG, Cune MS, Naeije M. Dental implants in patients with bruxing habits. J Oral Rehabil 2016; 33: 152-159.

4. Asutay F, Atalay Y, Asutay H, Acar AH. The evaluation of the clinical effects of botulinum toxin on nocturnal bruxism. Pain Res Manag 2017; 2017: 6264146.

5. Manfredini D, Lobbezoo F. Relationship between bruxism and temporomandibular disorders: a systematic review of literature from 1998 to 2008. Oral Med Oral Pathol Oral Radiol Endodontol 2010; 109: 26-50.

6. Castro WH, Gomez RS, Da Silva Oliveira J, Moura MDG, Gomez RS. Botulinum toxin type A in the management of masseter muscle hypertrophy. J Oral Maxillofac Surg 2005; 63: 20-24.

7. Choe SW, Cho WI, Lee CK, Seo SJ. Effects of botulinum toxin type A on contouring of the lower face. Dermatologic Surg 2005; 31: 502-508.

8. Schiavo G, Matteoli M, Montecucco C. Neurotoxins affecting neuroexocytosis. Physiological Reviews. Am J Physiol 2000; 80: 717-766.

9. Blitzer A, Sulica L. Botulinum toxin: basic science and clinical uses in otolaryngology. Laryngoscope 2001; 111: 218-226.

10. Ventola CL. Social media and health care professionals: Benefits, risks, and best practices. P T 2014; 39: 491-520.

11. AlGhamdi KM, Moussa NA. Internet use by the public to search for health-related information. Int J Med Inform 2012; 81: 363-373.

12. Al-Silwadi FM, Gill DS, Petrie A, Cunningham SJ. Effect of social media in improving knowledge among patients having fixed appliance orthodontic treatment: a single-center randomized controlled trial. Am J Orthod Dentofac Orthop 2015; 148: 231237.

13. Kumar N, Pandey A, Venkatraman A, Garg N. Are video sharing Web sites a useful source of information on hypertension? J Am Soc Hypertens 2014; 8: 481-490.

14. Madathil KC, Rivera-Rodriguez AJ, Greenstein JS, Gramopadhye AK. Healthcare information on YouTube: a systematic review. Health Inform J 2015; 21: 173-194.

15. Menziletoglu D, Guler AY, Isik BK. Are YouTube videos related to dental implant useful for patient education? J Stomatol Oral Maxillofac Surg 2020; 2020: 10-13.

16. Yavuz MC, Buyuk SK, Genc E. Does YouTube ${ }^{\mathrm{TM}}$ offer high quality information? Evaluation of accelerated orthodontics videos. Ir J Med Sci 2020; 189: 505-509.

17. Yilmaz H, Aydin M. YouTube ${ }^{\mathrm{TM}}$ video content analysis on space maintainers. J Indian Soc Pedod Prev Dent 2020; 38: 34-40.
18. Pons-Fuster E, Ruiz Roca J, Tvarijonaviciute A, López-Jornet P. YouTube informatio about diabetes and oral healthcare. Odontology 2020; 108: 84-90.

19. Gas S, Zincir O, Bozkurt AP. Are YouTube videos useful for patients interested in Botulinum toxin for bruxism? J Oral Maxillofac Surg 2019; 77: 1776-1783.

20. Desai T, Shariff A, Dhingra V, Minhas D, Eure M, Kats M. Is content really king? An objective analysis of the public's response to medical videos on YouTube. PLoS One 2013; 8: 8-13.

21. Sampson M, Cumber J, Li C, Pound CM, Fuller A, Harrison D. A systematic review of methods for studying consumer health YouTube videos, with implications for systematic reviews. Peer J 2013; 1: e147.

22. Abukaraky A, Hamdan AA, Ameera MN, Nasief M, Hassona Y. Quality of YouTube TM videos on dental implants. Med Oral Patol Oral Cir Bucal 2018; 23: 463-468.

23. Small R, Hoang D. A practical guide to botulinum toxin procedures. Philadelphia: Wolters Kluwer/Lippincott Williams \& Wilkins Health; 2012. pp. 144.

24. De Maio M, Wu WTL, Goodman GJ, Monheit G. Facial assessment and injection guide for botulinum toxin and injectable hyaluronic acid fillers: focus on the lower face. Plast Reconstr Surg 2017; 140: 393-404.

25. Sundaram H, Liew S, Signorini M, et al. Global aesthetics consensus: hyaluronic acid fillers and Botulinum toxin type A - recommendations for combined treatment and optimizing outcomes in diverse patient populations. Plast Reconstr Surg 2016; 137: 961-971.

26. Singh AG, Singh S, Singh PP. YouTube for information on rheumatoid arthritis - a wakeup call? J Rheumatol 2012; 39: 899-903.

27. Carneiro B, Dizon DS. Prostate cancer social media: in YouTube we trust? Eur Urol 2019; 75: 568-569.

28. ElKarmi R, Hassona Y, Taimeh D, Scully C. YouTube as a source for parents' education on early childhood caries. Int J Paediatr Dent 2017; 27: 437-443.

29. Oremule B, Patel A, Orekoya O, Advani R, Bondin D. Quality and reliability of YouTube videos as a source of patient information on rhinoplasty. JAMA 2019; 145: 282-283.

30. Ozdede M, Peker I. Analysis of dentistry YouTube videos related to COVID-19. Braz Dent J 2020; 31: 392-398. 